\title{
Lower bounds for Jung constants of Orlicz sequence spaces
}

\author{
by Z. D. REN (Riverside, CA)
}

Abstract. A new lower bound for the Jung constant $J C\left(l^{(\Phi)}\right)$ of the Orlicz sequence space $l^{(\Phi)}$ defined by an $N$-function $\Phi$ is found. It is proved that if $l^{(\Phi)}$ is reflexive and the function $t \Phi^{\prime}(t) / \Phi(t)$ is increasing on $\left(0, \Phi^{-1}(1)\right]$, then

$$
J C\left(l^{(\Phi)}\right) \geq \frac{\Phi^{-1}(1 / 2)}{\Phi^{-1}(1)} .
$$

Examples in Section 3 show that the above estimate is better than in Zhang's paper (2003) in some cases and that the results given in Yan's paper (2004) are not accurate.

1. Introduction. We begin by recalling some definitions.

Definition 1 (Jung [6]). Let $X$ be a normed linear sqace. The number $J C(X)$ of $X$, called the Jung constant, is defined by

$$
J C(X)=\sup \left\{\frac{r(A, X)}{d(A)}: A \subset X \text { bounded and } d(A)>0\right\},
$$

where $r(A, X)=\inf \{\sup (\|x-z\|: x \in A): z \in X\}$ is the absolute Chebyshev radius of $A$ and $d(A)=\sup \{\|x-y\|: x, y \in A\}$, the diameter of $A$.

Clearly, $1 / 2 \leq J C(X) \leq 1$. Note that in Amir [1] and Franchetti [5], $2 J C(X)$ is called the Jung constant of $X$ and denoted by $J(X)$. Historical notes on the Jung constant can be found in Appell, Franchetti and Semenov [2] as well as in Rao and Ren [10, Ch. 4].

Definition 2 (Bynum [3]). The normal structure coefficient of a Banach space $X$ is defined as

$$
N(X)=\inf \left\{\frac{d(A)}{r(A)}: A \subset X \text { bounded, closed and convex, } d(A)>0\right\},
$$

where $r(A)=\inf \{\sup (\|x-y\|: x \in A): y \in \overline{\operatorname{co}}(A)\}$ is the relative Chebyshev radius of $A$ with respect to $\overline{\mathrm{co}}(A)$, and $\overline{\mathrm{co}}(A)$ is the closure of the convex hull of $\mathrm{A}$. 
It can be seen that $1 \leq N(X) \leq 2$. We say that $X$ has the uniform normal structure provided $N(X)>1$. If $X$ is infinite-dimensional, then $N(X) \leq \sqrt{2}$ (cf. Maluta [8]). If $X$ is nonreflexive, then $N(X)=1$.

Definition 3 (Maluta 8]). For a Banach space $X$, the self-Jung constant $\tilde{N}(X)$ is defined by $\tilde{N}(X)=\sup \{r(A) / d(A): A \subset X$ is as in the definition of $N(X)\}$.

In [1], $2 \tilde{N}(X)$ is called the self-Jung constant of $X$ and denoted by $J s(X)$. Since $\tilde{N}(X)=1 / N(X)$ and $J C(X) \leq \tilde{N}(X)$, for every Banach space $X$ we have

$$
1 / 2 \leq J C(X) \leq 1 / N(X) \leq 1,
$$

which is used in Sections 4 and 5.

Now we turn to $N$-functions and the corresponding Orlicz sequence spaces.

Definition 4. A function $\Phi: \mathbb{R} \rightarrow[0, \infty)$ is termed an $N$-function if (i) $\Phi$ is even and convex; (ii) $\Phi(u)=0 \Leftrightarrow u=0$, (iii) $\lim _{u \rightarrow 0} \Phi(u) / u=0$ and (iv) $\lim _{u \rightarrow \infty} \Phi(u) / u=\infty$ (cf. Krasnosel'skiǔ and Rutickiu [7, pp. 6-9] as well as Rao and Ren [10, p. 1]).

An $N$-function $\Phi(u)$ is said to satisfy the $\Delta_{2}$-condition for small $u$ [for large $u]$, in symbols $\Phi \in \Delta_{2}(0)\left[\Phi \in \Delta_{2}(\infty)\right]$, if there exist $K>2$ and $u_{0}>0$ such that $\Phi(2 u) \leq K \Phi(u)$ for $0 \leq u \leq u_{0}$ [for $u \geq u_{0}$ ]. $\Phi(u)$ is said to satisfy the $\nabla_{2}$-condition for small $u$ [for large $u$ ], written $\Phi \in \nabla_{2}(0)\left[\Phi \in \nabla_{2}(\infty)\right]$, if there are $C>1$ and $u_{0}>0$ such that $2 C \Phi(u) \leq \Phi(C u)$ for $0 \leq u \leq u_{0}$ [for $u \geq u_{0}$ ].

If $\Phi$ is an $N$-function, its complementary function $\Psi$ is given by $\Psi(v)=$ $\sup \{u|v|-\Phi(u): u \geq 0\}$, which is also an $N$-function.

It is known that $\Phi \in \nabla_{2}(0)\left[\Phi \in \nabla_{2}(\infty)\right] \Leftrightarrow \Psi \in \Delta_{2}(0)\left[\Psi \in \Delta_{2}(\infty)\right]$, where $\Psi$ is complementary to $\Phi$. For a pair $(\Phi, \Psi)$ of complementary $N$ functions the Orlicz sequence space $l^{\Phi}$ is defined as

$$
l^{\Phi}=\left\{x=(x(1), x(2), \ldots): \rho_{\Phi}(\lambda x)=\sum_{i=1}^{\infty} \Phi(\lambda x(i))<\infty \text { for some } \lambda>0\right\},
$$

on which the gauge norm (also called the Luxemburg norm in [7], 4] and [2]) and the Orlicz norm are given respectively by

$$
\|x\|_{(\Phi)}=\inf \left\{c>0: \rho_{\Phi}(x / c) \leq 1\right\}
$$

and

$$
\|x\|_{\Phi}=\sup \left\{\sum_{i=1}^{\infty}|x(i) y(i)|: \rho_{\Psi}(y) \leq 1\right\} .
$$


These norms are equivalent: $\|x\|_{(\Phi)} \leq\|x\|_{\Phi} \leq 2\|x\|_{(\Phi)}$. We set $l^{(\Phi)}=$ $\left(l^{\Phi},\|\cdot\|_{(\Phi)}\right)$ and $l^{\Phi}=\left(l^{\Phi},\|\cdot\|_{\Phi}\right)$. Of course, $l^{(\Phi)}$ and $l^{\Phi}$ are Banach spaces. It is known that for an $N$-function $\Phi, l^{(\Phi)}$ and $l^{\Phi}$ are reflexive $\Leftrightarrow \Phi \in$ $\Delta_{2}(0) \cap \nabla_{2}(0)$. The Jung constants of $l^{(\Phi)}$ and $l^{\Phi}$ have been studied before by Zhang [15. The main results in [15] can be summarized in the following two propositions.

Proposition 1. Let $\Phi$ be an $N$-function. Then

(i) $\Phi \notin \Delta_{2}(0) \cap \nabla_{2}(0) \Rightarrow J C\left(l^{(\Phi)}\right)=J C\left(l^{\Phi}\right)=1$;

(ii) $\Phi \in \Delta_{2}(0) \cap \nabla_{2}(0) \Rightarrow \max \left(J C\left(l^{(\Phi)}\right), J C\left(l^{\Phi}\right)\right)<1$.

The first part of Proposition 1 is nontrivial and the second part is a consequence of (1) and Theorem 3.1 in Chen [4, p. 107] (cf. [12] and [13]).

Proposition 2. Let $\Phi(u)=\int_{0}^{|u|} \varphi(t) d t$ and $\Psi(v)=\int_{0}^{|v|} \psi(s) d s$ denote a pair of complementary $N$-functions and let $\Phi \in \Delta_{2}(0) \cap \nabla_{2}(0)$. Then

$$
J C\left(l^{(\Phi)}\right) \geq \max \left(\frac{1}{2 \alpha_{\Phi}^{0}}, \beta_{\Phi}^{0}\right),
$$

where

$$
\alpha_{\Phi}^{0}=\liminf _{u \rightarrow 0} \frac{\Phi^{-1}(u)}{\Phi^{-1}(2 u)}, \quad \beta_{\Phi}^{0}=\limsup _{u \rightarrow 0} \frac{\Phi^{-1}(u)}{\Phi^{-1}(2 u)}
$$

and

$$
J C\left(l^{\Phi}\right) \geq \max \left(\beta_{\Psi}^{0}, \frac{1}{2 \alpha_{\Psi}^{0}}\right),
$$

where $\alpha_{\Psi}^{0}$ and $\beta_{\Psi}^{0}$ are defined similarly to (5).

Corollary 1. Let $(\Phi, \Psi)$ be as in Proposition 2. If the limit $C_{\Phi}^{0}=$ $\lim _{t \rightarrow 0} t \varphi(t) / \Phi(t)$ exists, then (4) and (6) reduce to

$$
\min \left(J C\left(l^{(\Phi)}\right), J C\left(l^{\Phi}\right)\right) \geq \max \left(2^{1 / C_{\Phi}^{0}-1}, 2^{-1 / C_{\Phi}^{0}}\right) .
$$

Proof. Note that $1<C_{\Phi}^{0}<\infty$ because $\Phi \in \Delta_{2}(0) \cap \nabla_{2}(0)$ (cf. Theorem 2 in [10, p. 3]). Hence (7) follows from Theorem 15 in [10, p. 11] and Corollary 9 in [10, p. 8].

We often use (7) in concrete examples, e.g. in [10, p. 144, Example 7] it is proved that $J C\left(l^{p}\right)=\max \left(2^{1 / p-1}, 2^{-1 / p}\right)$ for $1<p<\infty$; see also Example 4 in Section 3.

2. Results. We start by introducing spaces having a symmetric norm.

Definition 5. A basis $\left\{x_{i}\right\}$ of a real Banach space $X$ is called symmetric if it is equivalent to the basis $\left\{x_{\pi(i)}\right\}$ for every rearrangement $\pi$ of the set of natural numbers. An infinite-dimensional Banach space $X$ with a symmetric 
basis $\left\{x_{i}\right\}_{i=1}^{\infty}$ is said to have a symmetric norm if for every real sequence $\left\{a_{i}\right\}_{i=1}^{\infty}$ with $\sum_{i=1}^{\infty} a_{i} x_{i} \in X$,

$$
\left\|\sum_{i=1}^{\infty} a_{i} x_{i}\right\|_{X}=\left\|\sum_{i=1}^{\infty} \theta_{i} a_{i} x_{\pi(i)}\right\|_{X}
$$

for all choices of signs $\theta_{i}= \pm 1$ and all permulations $\pi$ of the natural numbers.

The following lemma will be used.

Lemma 1 (Franchetti [5, Lemma 2]). Let $X$ be an infinite-dimensional Banach space with symmetric basis $\left\{e_{i}\right\}_{i=1}^{\infty}$ and symmetric norm. Define $f: \mathbb{N}=\{1,2, \ldots\} \rightarrow \mathbb{R}^{+}$by $f(k, X)=\left\|\sum_{i=1}^{k} e_{i}\right\|_{X}$. Then

$$
J C(X) \geq \sup _{k \in \mathbb{N}} \frac{f(k, X)}{f(2 k, X)} .
$$

TheOrem 1. Let $\Phi(u)=\int_{0}^{|u|} \varphi(t) d t$ and $\Psi(v)=\int_{0}^{|v|} \psi(s) d s$ denote $a$ pair of complementary $N$-functions and let $\Phi \in \Delta_{2}(0) \cap \nabla_{2}(0)$. Define

$$
\beta_{\Phi}^{\prime}=\sup _{k \in \mathbb{N}} \frac{\Phi^{-1}(1 / 2 k)}{\Phi^{-1}(1 / k)}, \quad \alpha_{\Psi}^{\prime}=\inf _{k \in \mathbb{N}} \frac{\Psi^{-1}(1 / 2 k)}{\Psi^{-1}(1 / k)} .
$$

Then

$$
\begin{aligned}
J C\left(l^{(\Phi)}\right) & \geq \beta_{\Phi}^{\prime}, \\
J C\left(l^{\Phi}\right) & \geq \frac{1}{2 \alpha_{\Psi}^{\prime}} .
\end{aligned}
$$

Proof. Let $e_{1}=(1,0,0, \ldots), e_{2}=(0,1,0, \ldots)$, etc. The canonical basis $\left\{e_{i}\right\}_{i=1}^{\infty}$ is symmetric in $l^{(\Phi)}$ as well as in $l^{\Phi}$ since $\Phi \in \Delta_{2}(0)$ (cf. [4, p. 47]). The norms (2) and (3) are symmetric. It can be seen from (2) that

$$
f\left(k, l^{(\Phi)}\right)=\left\|\sum_{i=1}^{k} e_{i}\right\|_{(\Phi)}=\inf \{c>0: k \Phi(1 / c) \leq 1\}=\frac{1}{\Phi^{-1}(1 / k)} .
$$

Thus (10) follows from (8), (12) and (9). Let $\lambda_{0}$ satisfy the equation

$$
1=\rho_{\Psi}\left[\varphi\left(\lambda_{0} \sum_{i=1}^{k} e_{i}\right)\right]=k \Psi\left[\varphi\left(\lambda_{0}\right)\right] .
$$

Then $\varphi\left(\lambda_{0}\right)=\Psi^{-1}(1 / k)$. By Proposition 14 in [9, p. 70] we have

$$
f\left(k, l^{\Phi}\right)=\left\|\sum_{i=1}^{k} e_{i}\right\|_{\Phi}=\left\langle\sum_{i=1}^{k} e_{i}, \varphi\left(\lambda_{0} \sum_{i=1}^{k} e_{i}\right)\right\rangle=k \varphi\left(\lambda_{0}\right)=k \Psi^{-1}(1 / k) .
$$

Finally, (11) follows from (8), (13) and (9). 
Theorem 2. Let $\Phi(u)=\int_{0}^{|u|} \varphi(t) d t$ and $\Psi(v)=\int_{0}^{|v|} \psi(s) d s$ be as in Theorem 1 and let $F_{\Phi}(t)=t \varphi(t) / \Phi(t), t>0$. Assume that $\varphi$ is continuous and strictly increasing on $\left[0, \max \left\{\Phi^{-1}(1), \psi\left[\Psi^{-1}(1)\right]\right\}\right]$.

(i) If $F_{\Phi}(t)$ is increasing on $\left(0, \Phi^{-1}(1)\right]$, then

$$
J C\left(l^{(\Phi)}\right) \geq \max \left(2^{1 / C_{\Phi}^{0}-1}, \frac{\Phi^{-1}(1 / 2)}{\Phi^{-1}(1)}\right)
$$

where

$$
C_{\Phi}^{0}=\lim _{t \rightarrow 0} F_{\Phi}(t) .
$$

(ii) If $F_{\Phi}(t)$ is increasing on $\left(0, \psi\left[\Psi^{-1}(1)\right]\right]$, then

$$
J C\left(l^{\Phi}\right) \geq \max \left(2^{1 / C_{\Phi}^{0}-1}, \frac{\Psi^{-1}(1)}{2 \Psi^{-1}(1 / 2)}\right) .
$$

Proof. (i) Since $F_{\Phi}(t)$ is increasing on $\left(0, \Phi^{-1}(1)\right]$ and $\Phi \in \Delta_{2}(0) \cap \nabla_{2}(0)$, the limit $C_{\Phi}^{0}$ exists and $1<C_{\Phi}^{0}<\infty$ as noted before. Further, the function $G_{\Phi}(u)=\Phi^{-1}(u) / \Phi^{-1}(2 u)$ for $u>0$ is increasing on $(0,1 / 2$ ] (cf. [10, p. 93]) and $\gamma_{\Phi}^{0}=\lim _{u \rightarrow 0} G_{\Phi}(u)$ exists. It follows from (9) and Corollary 9 in [10, p. 8] that

$$
\beta_{\Phi}^{\prime}=\frac{\Phi^{-1}(1 / 2)}{\Phi^{-1}(1)}=G_{\Phi}(1 / 2) \geq \lim _{u \rightarrow 0} G_{\Phi}(u)=\gamma_{\Phi}^{0}=2^{-1 / C_{\Phi}^{0}},
$$

which proves (14) by (10) and (7).

(ii) Recall that the Young inequality $|u v| \leq \Phi(u)+\Psi(v)$ becomes an equality when $|v|=\varphi(|u|)$ or $|u|=\psi(|v|)$ (see [9, p. 10]). Note that $\varphi(t)$ is strictly increasing on $\left(0, \psi\left[\Psi^{-1}(1)\right]\right]$ if and only of $\psi(s)$ is continuous on $\left(0, \Psi^{-1}(1)\right]$. By letting $s=\varphi(t), 0<t \leq \psi\left[\Psi^{-1}(1)\right]$, we see that $t=\psi(s)$ and

or

$$
F_{\Psi}(s)=\frac{s \psi(s)}{\Psi(s)}=\frac{t \varphi(t)}{t \varphi(t)-\Phi(t)}=\frac{F_{\Phi}(t)}{F_{\Phi}(t)-1},
$$

$$
\frac{1}{F_{\Phi}(t)}+\frac{1}{F_{\Phi}(s)}=1, \quad 0<s=\varphi(t) \leq \Psi^{-1}(1),
$$

which implies

$$
\frac{1}{C_{\Phi}^{0}}+\frac{1}{C_{\Psi}^{0}}=1
$$

where $C_{\Psi}^{0}=\lim _{s \rightarrow 0} F_{\Psi}(s)$. By the assumption on $F_{\Phi}(t)$ and $(17), F_{\Psi}(s)$ is decreasing on $\left(0, \Psi^{-1}(1)\right]$ so that $G_{\Psi}(v)=\Psi^{-1}(v) / \Psi^{-1}(2 v)$ is decreasing on $(0,1 / 2]$ and $\gamma_{\Psi}^{0}=\lim _{v \rightarrow 0} G_{\Psi}(v)$ exists. Finally,

$$
2 \alpha_{\Psi}^{\prime}=\frac{2 \Psi^{-1}(1 / 2)}{\Psi^{-1}(1)}=2 G_{\Psi}(1 / 2) \leq 2 \alpha_{\Psi}^{0}=2 \gamma_{\Psi}^{0}=2^{1-1 / C_{\Psi}^{0}}
$$

so that (16) follows from (11), (7) and (18). 
3. Examples. To illustrate Theorems 1 and 2 we present some examples, that were considered by Zhang [15] and Yan [14], except Example 4.

EXAmple 1. Consider the following pair of complementary $N$-functions:

$$
\Phi(u)=e^{|u|}-|u|-1, \quad \Psi(v)=(1+|v|) \ln (1+|v|)-|v| .
$$

In [10, p. 9] it was proved that $F_{\Phi}(t)\left(=t \Phi^{\prime}(t) / \Phi(t)\right)$ is increasing on $(0, \infty)$ and $C_{\Phi}^{0}=\lim _{t \rightarrow 0} F_{\Phi}(t)=2$, so that $\Phi \in \Delta_{2}(0) \cap \nabla_{2}(0)$. By Theorem 2 we obtain

$$
J C\left(l^{(\Phi)}\right) \geq \frac{\Phi^{-1}(1 / 2)}{\Phi^{-1}(1)} \approx 0.7483, \quad J C\left(l^{\Phi}\right) \geq \frac{\Psi^{-1}(1)}{2 \Psi^{-1}(1 / 2)} \approx 0.7435,
$$

which refine the inequalities $J C\left(l^{(\Phi)}\right), J C\left(l^{\Phi}\right) \geq 1 / \sqrt{2}$ in [15, Example 2.12]. In fact, Franchetti [5] proved that $J C(X) \geq 1 / \sqrt{2}$ for any infinitedimensional Banach space $X$ with a symmetric norm (see also [2, Theorem 1]).

EXAmple 2. For the space $l^{\left(\Phi_{p}\right)}$ generated by the $N$-function

$$
\Phi_{p}(u)=e^{|u|^{p}}-1, \quad 1<p<\infty,
$$

we have

$$
J C\left(l^{\left(\Phi_{p}\right)}\right) \geq\left(\frac{\ln 3-\ln 2}{\ln 2}\right)^{1 / p}>\max \left(2^{1 / p-1}, 2^{-1 / p}\right)
$$

if $1+a<p<\infty$, where

$$
a=\frac{1}{\ln 2} \ln \left(\frac{\ln 2}{\ln 3-\ln 2}\right) \approx 0.7736 .
$$

Proof. The function $F_{\Phi_{p}}(t)=p t^{p} e^{t^{p}} /\left(e^{t^{p}}-1\right)$ is increasing on $(0, \infty)$ and $C_{\Phi_{p}}^{0}=\lim _{t \rightarrow 0} F_{\Phi_{p}}(t)=p$, so that $\Phi_{p} \in \Delta_{2}(0) \cap \nabla_{2}(0)$. We first consider $2 \leq p<\infty$. Since $\Phi_{p}^{-1}(u)=[\ln (1+u)]^{1 / p}$ for $u>0$, from (14) in Theorem 2 we have

$$
J C\left(l^{\left(\Phi_{p}\right)}\right) \geq \frac{\Phi_{p}^{-1}(1 / 2)}{\Phi_{p}^{-1}(1)}>2^{-1 / p} \geq 2^{1 / p-1}, \quad 2 \leq p<\infty,
$$

which proves $(20)$ when $2 \leq p<\infty$. Next we consider $1<p<2$. It can be seen that $\Phi_{p}^{-1}(1 / 2) / \Phi_{p}^{-1}(1)>2^{1 / p-1}$ if and only if $1+a<p<2$, where $a$ is given by (21). Thus,

$$
J C\left(l^{\left(\Phi_{p}\right)}\right) \geq \frac{\Phi_{p}^{-1}(1 / 2)}{\Phi_{p}^{-1}(1)}>2^{1 / p-1}>2^{-1 / p}, \quad 1+a<p<2,
$$

which completes the proof of (20).

REMARK 1. The estimate (20) improves the estimate in [15, Example 2.11] when $1+a<p<\infty$. The estimate (22) shows that the exact 
value for $J C\left(l^{\left(\Phi_{p}\right)}\right)$ given in [14, Example 2.7], $J C\left(l^{\left(\Phi_{p}\right)}\right)=2^{1 / p-1}, 1<p<2$, is impossible at least when $1.7736 \approx 1+a<p<2$.

Example 3. Consider the $N$-function

$$
M_{p}(u)=|u|^{2 p}+2|u|^{p}, \quad 1<p<\infty .
$$

Then

$$
J C\left(l^{\left(M_{p}\right)}\right) \geq\left(\frac{\sqrt{3}-\sqrt{2}}{2-\sqrt{2}}\right)^{1 / p}>\max \left(2^{1 / p-1}, 2^{-1 / p}\right)
$$

if $1+b<p<\infty$, where

$$
b=\frac{1}{\ln 2} \ln \left(\frac{2-\sqrt{2}}{\sqrt{3}-\sqrt{2}}\right) \approx 0.8812 .
$$

Proof. $F_{M_{p}}(t)$ is increasing on $(0, \infty)$ from $p$ to $2 p$ and $C_{M_{p}}^{0}=p$, so that $M_{p} \in \Delta_{2}(0) \cap \nabla_{2}(0)$. The inverse of $M_{p}$ is $M_{p}^{-1}(u)=(\sqrt{1+u}-1)^{1 / p}, u>0$. Thus, the proof is similar to that of Example 2.

REMARK 2. The estimate (24) and (25) show that the exact value for $J C\left(l^{\left(M_{p}\right)}\right)$ in [14, Example 2.6] is not true when $1+b<p<2$.

Let us make a comparison between Corollary 1 and Theorem 2 when $C_{\Phi}^{0}$ exists to estimate the lower bounds of $J C\left(l^{(\Phi)}\right)$ and $J C\left(l^{\Phi}\right)$. If $F_{\Phi}(t)$ is increasing on $\left(0, \Phi^{-1}(1)\right]$ or on $\left(0, \psi\left[\Psi^{-1}(1)\right]\right]$, then Theorem 2 is an improvement of Corollary 1, as shown by Examples 1-3. On the other hand, Theorem 1 is not better than Proposition 2 if $F_{\Phi}(t)$ is decreasing.

ExAmPle 4. Let $M_{p, \lambda}(u)=|u|^{p}[\ln (1+|u|)]^{r}$ with $1 \leq p<\infty$ and $0<\lambda<\infty$, which is considered in [2, p. 184] when $p=2$ and $\lambda \geq 0$. The function $F_{M_{p, \lambda}}(t)=p+\{\lambda t /[(1+t) \ln (1+t)]\}$ is decreasing on $(0, \infty)$ and $C_{M_{p, \lambda}}^{0}=p+\lambda>1$, so that $M_{p, \lambda} \in \Delta_{2}(0) \cap \nabla_{2}(0)$. It can be seen that $\beta_{M_{p, \lambda}}^{\prime} \leq \lim _{u \rightarrow 0} G_{M_{p, \lambda}}(u)=\beta_{M_{p, \lambda}}^{0}$. Therefore, Proposition 2 improves Theorem 1. Finally, from Corollary 1 we obtain

$$
\min \left(J C\left(l^{\left(M_{p, \lambda}\right)}\right), J C\left(l^{M_{p, \lambda}}\right)\right) \geq \max \left(2^{1 / p+\lambda-1}, 2^{-1 / p+\lambda}\right)=J C\left(l^{p+\lambda}\right)
$$

(see the end of Section 1 for $J C\left(l^{p}\right)$ ).

4. Intermediate Orlicz sequence spaces. Now we turn to certain intermediate $N$-functions and the corresponding intermediate Orlicz sequence spaces.

Definition 6. Let $\Phi$ be an $N$-function and let $\Phi_{0}(u)=u^{2}$. For each $0<s \leq 1$ we define an intermediate $N$-function $\Phi_{s}$ between $\Phi$ and $\Phi_{0}$ at $s$ to be the inverse of

$$
\Phi_{s}^{-1}(u)=\left[\Phi^{-1}(u)\right]^{1-s}\left[\Phi_{0}^{-1}(u)\right]^{s}=\left[\Phi^{-1}(u)\right]^{1-s}(\sqrt{u})^{s}, \quad u \geq 0 .
$$


The corresponding Orlicz sequence space $l^{\left(\Phi_{s}\right)}\left[l^{\Phi_{s}}\right]$ is called an intermediate space between $l^{(\Phi)}\left[l^{\Phi}\right]$ and $l^{2}$ at $s \in(0,1]$ in the sense of Calderón (cf. [9, p. 223]).

The author [11] proved that $\Phi_{s} \in \Delta_{2} \cap \nabla_{2}$ if $0<s \leq 1$, where $\Delta_{2}=$ $\Delta_{2}(0) \cap \Delta_{2}(\infty)$ and $\nabla_{2}=\nabla_{2}(0) \cap \nabla_{2}(\infty)$, that is, $\Phi_{s}(u)$ satisfies both the

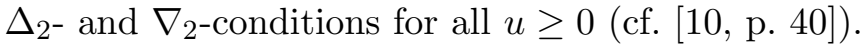

The following result enables us to find the exact values of $J C\left(l^{\left(\Phi_{s}\right)}\right)$ and $J C\left(l^{\Phi_{s}}\right)$ as well as the normal structure coefficients $N\left(l^{\left(\Phi_{s}\right)}\right)$ and $N\left(l^{\Phi_{s}}\right)$.

Lemma 2 (Ren [11]). Let $\Phi$ be an $N$-function and let $\Phi_{s}$ be the inverse of (26). Then for every $s \in(0,1]$ we have

$$
2^{s / 2} \leq \min \left(N\left(l^{\left(\Phi_{s}\right)}\right), N\left(l^{\Phi_{s}}\right)\right) .
$$

The proof of (27) can be found also in [10, p. 85]. The main result of this section is given by:

TheOREM 3. Let $\Phi$ and $\Phi_{s}$ be as in Lemma 2. Then

$$
\begin{array}{r}
\max \left(\beta_{\Phi_{s}}^{\prime}, \frac{1}{2 \alpha_{\Phi_{s}}^{0}}, \beta_{\Phi_{s}}^{0}\right) \leq J C\left(l^{\left(\Phi_{s}\right)}\right) \leq 2^{-s / 2}, \\
\max \left(\frac{1}{2 \alpha_{\Psi_{s}^{+}}^{\prime}}, \frac{1}{2 \alpha_{\Phi_{s}}^{0}}, \beta_{\Phi_{s}}^{0}\right) \leq J C\left(l^{\Phi_{s}}\right) \leq 2^{-s / 2},
\end{array}
$$

where $\Psi_{s}^{+}$is complementary to $\Phi_{s}$. In particular, if $\Phi \notin \Delta_{2}(0) \cap \nabla_{2}(0)$, then $J C\left(l^{\left(\Phi_{s}\right)}\right)=2^{-s / 2}=J C\left(l^{\Phi_{s}}\right)$.

Proof. (28) and (29) follow from (27), (1), Proposition 2, Theorem 1 and the fact that $2 \alpha_{\Phi_{s}}^{0} \beta_{\Psi_{s}^{+}}^{0}=1=2 \alpha_{\Psi_{s}^{+}}^{0} \beta_{\Phi_{s}}^{0}$ (cf. [10, p. 15]). The proof of the second part is the same as that of Theorem 9 in [10, p. 145].

Remark 3. Theorem 3 partly refines Theorem 6 in [10, p. 144].

For computing the exact value of the Jung constant in a special Banach space we now present a simple example. Consider the $N$-function

$$
M_{p}(u)=|u|^{p} e^{-1 /|u|}, \quad 1<p<\infty .
$$

Its inverse $M_{p}^{-1}$ on $[0, \infty)$ is not obtained explicitly. Note that

$$
C_{M_{p}}^{0}=\lim _{t \rightarrow 0} t M_{p}^{\prime}(t) / M_{p}(t)=\lim _{t \rightarrow 0}(p+1 / t)=\infty,
$$

so that $M_{p} \notin \Delta_{2}(0)$. Let $\Phi_{s}$ be the inverse of the function $\Phi_{s}^{-1}(u)=$ $\left[M_{p}^{-1}(u)\right]^{1-s}(\sqrt{u})^{s}, u \geq 0$ and $0<s \leq 1$. By the second part of Theorem 3 we obtain the exact values $J C\left(l^{\left(\Phi_{s}\right)}\right)=2^{-s / 2}=J C\left(l^{\Phi_{s}}\right)$.

For the upper bounds of the normal structure coefficients $N\left(l^{(\Phi)}\right)$ and $N\left(l^{\Phi}\right)$ we have the following. 
Proposition 3. Let $(\Phi, \Psi)$ be a pair of complementary $N$-functions with $\Phi \in \Delta_{2}(0) \cap \nabla_{2}(0)$. Then

$$
\begin{gathered}
N\left(l^{(\Phi)}\right) \leq \min \left(2 \alpha_{\Phi}^{0}, \frac{1}{\beta_{\Phi}^{0}}, \frac{1}{\beta_{\Phi}^{\prime}}\right), \\
N\left(l^{\Phi}\right) \leq \min \left(2 \alpha_{\Phi}^{0}, \frac{1}{\beta_{\Phi}^{0}}, 2 \alpha_{\Psi}^{\prime}\right),
\end{gathered}
$$

where $\alpha_{\Phi}^{0}, \beta_{\Phi}^{0}, \beta_{\Phi}^{\prime}$ and $\alpha_{\Psi}^{\prime}$ are as in (5) and (9).

Proof. In [10, p. 108] it was proved that

$$
\max \left(N\left(l^{(\Phi)}\right), N\left(l^{\Phi}\right)\right) \leq \min \left(2 \alpha_{\Phi}^{0}, \frac{1}{\beta_{\Phi}^{0}}\right) .
$$

It should be noted that (32) can also be deduced immediately from Proposition 2 and (1). By Theorem 1 we get

$$
N\left(l^{(\Phi)}\right) \leq 1 / \beta_{\Phi}^{\prime}, \quad N\left(l^{\Phi}\right) \leq 2 \alpha_{\Psi}^{\prime} .
$$

Finally, (30) and (31) follow from (32) and (33).

Corollary 2. Let $(\Phi, \Psi)$ and $F_{\Phi}(t)$ be as in Theorem 2.

(i) If $F_{\Phi}(t)$ is increasing on $\left(0, \Phi^{-1}(1)\right]$, then

$$
N\left(l^{(\Phi)}\right) \leq \min \left(2^{1-1 / C_{\Phi}^{0}}, \frac{\Phi^{-1}(1)}{\Phi^{-1}(1 / 2)}\right) .
$$

(ii) If $F_{\Phi}(t)$ is increasing on $\left(0, \psi\left[\Psi^{-1}(1)\right]\right]$, then

$$
N\left(l^{\Phi}\right) \leq \min \left(2^{1-1 / C_{\Phi}^{0}}, \frac{2 \Psi^{-1}(1 / 2)}{\Psi^{-1}(1)}\right) .
$$

By Lemma 2 and Proposition 3 we get the desired result.

Theorem 4. Let $\Phi$ and $\Phi_{s}$ be as in Lemma 2. Then

$$
\begin{aligned}
& 2^{s / 2} \leq N\left(l^{\left(\Phi_{s}\right)}\right) \leq \min \left(2 \alpha_{\Phi_{s}}^{0}, \frac{1}{\beta_{\Phi_{s}}^{0}}, \frac{1}{\beta_{\Phi_{s}}^{\prime}}\right), \\
& 2^{s / 2} \leq N\left(l^{\Phi_{s}}\right) \leq \min \left(2 \alpha_{\Phi_{s}}^{0}, \frac{1}{\beta_{\Phi_{s}}^{0}}, 2 \alpha_{\Psi_{s}^{+}}^{\prime}\right) .
\end{aligned}
$$

In particular, if $\Phi \notin \Delta_{2}(0) \cap \nabla_{2}(0)$, we get $N\left(l^{\left(\Phi_{s}\right)}\right)=2^{s / 2}=N\left(l^{\Phi_{s}}\right)$.

REMARK 4. Theorem 4 partly refines Theorem 8 in [10, p. 114].

5. Supplements to examples. Now we apply Corollary 2, Theorems 3 and 4 to Examples 1-3 in Section 3. 
Example 5. Let $(\Phi, \Psi)$ be as in Example 1. By (34) and (35) we obtain

$$
N\left(l^{(\Phi)}\right) \leq \frac{\Phi^{-1}(1)}{\Phi^{-1}(1 / 2)} \approx 1.3364, \quad N\left(l^{\Phi}\right) \leq \frac{2 \Psi^{-1}(1 / 2)}{\Psi^{-1}(1)} \approx 1.3450 .
$$

Before studying the next two examples we have to prove the following.

Lemma 3. Let $M(u)=\int_{0}^{|u|} p(t) d t$ be an $N$-function and let $\Phi_{s}(u)=$ $\int_{0}^{|u|} \varphi_{s}(t) d t$ be the inverse of $\Phi_{s}^{-1}(u)=\left[M^{-1}(u)\right]^{1-s}(\sqrt{u})^{s}, u \geq 0$ and $0<$ $s \leq 1$. Consider $F_{M}(t)=t p(t) / M(t), t>0$, and $F_{\Phi_{s}}(t)=t \varphi_{s}(t) / \Phi_{s}(t)$, $t>0$. Then $F_{M}(t)$ is increasing on $\left(0, M^{-1}(1)\right]$ if and only if $F_{\Phi_{s}}\left(t_{s}\right)$ is increasing on $\left(0, \Phi_{s}^{-1}(1)\right]$.

Proof. Since $\ln \Phi_{s}^{-1}(u)=(1-s) \ln M^{-1}(u)+(s / 2) \ln u$ for $u>0$ we get by differentiation

$$
\frac{1}{\Phi_{s}^{-1}(u) \varphi_{s}\left[\Phi_{s}^{-1}(u)\right]}=\frac{1-s}{M^{-1}(u) p\left[M^{-1}(u)\right]}+\frac{s}{2 u},
$$

so that

$$
\frac{\Phi_{s}\left[\Phi_{s}^{-1}(u)\right]}{\Phi_{s}^{-1}(u) \varphi_{s}\left[\Phi_{s}^{-1}(u)\right]}=\frac{(1-s) M\left[M^{-1}(u)\right]}{M^{-1}(u) p\left[M^{-1}(u)\right]}+\frac{s}{2}, \quad u>0 .
$$

By letting $u=M(t)$ and $t_{s}=\Phi_{s}^{-1}(u)=\Phi_{s}^{-1}[M(t)]=t^{1-s}[M(t)]^{s / 2}$ in the above, we see that $0<t_{s} \leq\left[M^{-1}(1)\right]^{1-s}=\Phi_{s}^{-1}(1)$ if $0<t \leq M^{-1}(1)$ and that

$$
\frac{1}{F_{\Phi_{s}}\left(t_{s}\right)}=\frac{1-s}{F_{M}(t)}+\frac{s}{2}
$$

which proves the lemma.

Example 6 . Let $\Phi_{p}$ be given by (19) in Example 2 .

(i) It follows from (34) in Corollary 2 that

$$
N\left(l^{\left(\Phi_{p}\right)}\right) \leq\left(\frac{\ln 2}{\ln 3-\ln 2}\right)^{1 / p}<\min \left(2^{1-1 / p}, 2^{1 / p}\right)
$$

if $1+a<p<\infty$, where $a$ is in (21).

(ii) Let $\Phi_{s}(u)$ be the intermediate $N$-function between $\Phi_{p}(u)$ and $u^{2}$ at $s \in(0,1]$, i.e.,

$$
\Phi_{s}^{-1}(u)=[\ln (1+u)]^{(1-s) / p}(\sqrt{u})^{s}, \quad u \geq 0 .
$$

By Lemma $3, F_{\Phi_{s}}(t)$ is increasing on $\left(0, \Phi_{s}^{-1}(1)\right]$ and

$$
\beta_{\Phi_{s}}^{\prime}=\frac{\Phi_{s}^{-1}(1 / 2)}{\Phi_{s}^{-1}(1)}=\left(\beta_{\Phi_{p}}^{\prime}\right)^{1-s}\left(\frac{1}{\sqrt{2}}\right)^{s} .
$$


From Theorem 3 and Theorem 2 for $1+a<p<\infty$ with $a$ being from (21), we have

$$
\begin{aligned}
2^{-s / 2} \geq J C\left(l^{\left(\Phi_{s}\right)}\right) \geq \beta_{\Phi_{s}}^{\prime} & =2^{-s / 2}\left(\frac{\ln 3-\ln 2}{\ln 2}\right)^{(1-s) / p} \\
& >\max \left(2^{(1-s) / p+s / 2-1}, 2^{(s-1) / p-s / 2}\right) .
\end{aligned}
$$

(iii) Let $\Phi_{s}$ be the inverse of (38). By Theorem 4 we have $2^{s / 2} \leq N\left(l^{\left(\Phi_{s}\right)}\right)$ $\leq 1 / \beta_{\Phi_{s}}^{\prime}$ if $1+a<p<\infty$.

REMARK 5. (39) shows that the exact value for $J C\left(l^{\left(\Phi_{s}\right)}\right)$ in [14, Example 2.7, equality (20)] is not valid when $1+a<p<2$.

Also, (39) is an improvement of Example 8 in [10, p. 144].

(iii) in Example 6 refines Example 9 in [10, p. 115].

ExAmple 7. Let $M_{p}$ be given by (23) of Example 3. Similarly to Example 6 , we have the following assertions:

(i) Let $1+b<p<\infty$ with $b$ as in (25). Then

$$
N\left(l^{\left(M_{p}\right)}\right) \leq \frac{1}{\beta_{M_{p}}^{\prime}}=\left(\frac{2-\sqrt{2}}{\sqrt{3}-\sqrt{2}}\right)^{1 / p}<\min \left(2^{1-1 / p}, 2^{1 / p}\right) .
$$

(ii) Let $M_{s}$ be the inverse of $M_{s}^{-1}(u)=(\sqrt{1+u}-1)^{(1-s) / p}(\sqrt{u})^{s}, u \geq 0$ and $0<s \leq 1$. Then note that

$$
\beta_{M_{s}}^{\prime}=\left(\beta_{M_{p}}^{\prime}\right)^{1-s}\left(\frac{1}{2}\right)^{s / 2}=2^{-s / 2}\left(\frac{\sqrt{3}-\sqrt{2}}{2-\sqrt{2}}\right)^{(1-s) / p}
$$

and that (37) implies

$$
\frac{1}{C_{M_{s}}^{0}}=\frac{1-s}{C_{M_{p}}^{0}}+\frac{s}{2}=\frac{1-s}{p}+\frac{s}{2} .
$$

Moreover, it follows from Theorems 2 and 3 that for $1+b<p<\infty$,

$$
2^{-s / 2} \geq J C\left(l^{\left(M_{s}\right)}\right) \geq \beta_{M_{s}}^{\prime}>\max \left(2^{1 / C_{M_{s}}^{0}-1}, 2^{\left.-1 / C_{M_{s}}^{0}\right) .}\right.
$$

(iii) Let $M_{s}$ be as in (ii). Then $2^{s / 2} \leq N\left(l^{\left(M_{s}\right)}\right) \leq 1 / \beta_{M_{s}}^{\prime}$ whenever $1+b<p<\infty$.

REMARK 6. (40) shows that the exact value in [14, Example 2.6] is not true when $1.8821 \approx 1+b<p<2$. Also, (40) is an improvement of Example 3.7 in [15, p. 43].

Acknowledgments. The author would like to thank Professor C. Franchetti for sending his article [5]. Also, the author is grateful to Professor M. M. Rao for his helpful comments about the first draft. 


\section{References}

[1] D. Amir, On Jung's constant and related constants in normed linear spaces, Pacific J. Math. 118 (1985), 1-15.

[2] J. Appell, C. Franchetti and E. M. Semenov, Estimates for the Jung constant in Banach lattices, Israel J. Math. 116 (2000), 171-187.

[3] W. L. Bynum, Normal structure coefficients for Banach spaces, Pacific J. Math. 86 (1980), 427-435.

[4] S. T. Chen, Geometry of Orlicz Spaces, Dissertationes Math. 356 (1996).

[5] C. Franchetti, On the Jung constant in Banach spaces with a symmetric norm, in: Israel Math. Conf. Proc. 4, Bar-Ilan Univ., 1991, 129-134.

[6] H. W. E. Jung, Ueber die kleinste Kugel, die eine räumliche Figur einschliesst, J. Reine Angew. Math. 123 (1901), 241-257.

[7] M. A. Krasnosel'skiı̌ and Ya. B. Rutickiı̌, Convex Functions and Orlicz Spaces, Noordhoff, Groningen, 1961.

[8] E. Maluta, Uniformly normal structure and related coefficients, Pacific J. Math. 111 (1984), 357-369.

[9] M. M. Rao and Z. D. Ren, Theory of Orlicz Spaces, Dekker, New York, 1991.

[10] -, 一, Applications of Orlicz Spaces, Dekker, New York, 2002.

[11] Z. D. Ren, Some geometric coefficients in Orlicz function spaces, in: Lecture Notes in Pure Appl. Math. 175, Dekker, 1996, 391-404.

[12] S. Tian, X. Q. Liu and C. W. Xia, On the uniformly normal structure of Orlicz sequence spaces with Luxemburg norm, J. Harbin Univ. Sci. Tech. 19 (1995), no. 5, 87-94 (in Chinese); MR 99f:46028.

[13] - , - - - On the uniformly normal structure of Orlicz sequence spaces with Orlicz norm, J. Heilongjiang Univ. (N. S.) 13 (1996), no. 2, 22-27 (in Chinese); MR 99f:46028.

[14] Y. Q. Yan, On the exact value of Jung constants of Orlicz sequence spaces, Collect. Math. 55 (2004), 163-170.

[15] T. Zhang, Jung constants of Orlicz sequence spaces, Ann. Polon. Math. 81 (2003), $25-45$.

Z. D. Ren

Department of Mathematics

University of California

Riverside, CA 92521, U.S.A.

E-mail: jqiu98@hotmail.com

Received 26.11.2008

and in final form 26.7.2009 